\title{
Research on Fuzzy Multi-objective Programming based on Effect Probability
}

\author{
Fachao Li, Jingduo Jie \\ School of Ecnomics and Management, Hebei University of Science and Technology, Shijiazhuang, \\ 050018, China
}

Keywords: FMO-EP, effect probability, multi-objective programming, fuzzy number, decision.

\begin{abstract}
Crisp multi-objective programming has been applied successfully in many fields. But in real applications, the decision environment often exists randomness and the objectives are fuzzy. So it is significant and valuable to establish a fuzzy multi-objective programming model under random environment. In this paper, we use fuzzy number to represent the fuzzy events and fuzzy objectives. Firstly, the general formula of effect probability based on continuous distributions is given combined with effect theory, and a special one based on normal distribution is further given. Secondly, an effect probability-based fuzzy multi-objective programming (abbreviated as FMO-EP) model is proposed by regarding fuzzy objectives as fuzzy events. Finally, the effectiveness and interpretability of FMO-EP is proved through a case.
\end{abstract}

\section{Introduction}

Probability theory as a tool dealing with the randomness has successfully applied to many fields. The random events are fail to describe the subjective uncertain of random events in real life because it is crisp (for example, the event of "the profit is not less than $a$ thousand yuan as far as possible" and "the time of production equipment is not more than $b$ hours as far as possible" in the multiobjective programming, they are both fuzzy). For this, Zadeh [1] firstly proposed a probability model of fuzzy events on the basis of regarding membership degree as the coefficient, in 1968. In 2008, Chen etc. [2] proposed a probability model of fuzzy events based on decomposition theorem of fuzzy sets and the probability of different level sets. In this model, integral is the comprehensive operator. But the effect of elements in different status was not taken consideration into the decisionmaking process.

Multi-objective programming is a common method to deal with the multi- objective problems in certain environment. But the decision environment is usually random and fuzzy. [3, 4] proposed a new multi-objective programming models solving the randomness and fuzziness in uncertain environment, respectively. However there is no effective multi-objective programming model for the multi-objective problems whose objective coefficients are random variables and objectives are fuzzy.

Triangular fuzzy number is a common tool representing fuzzy information because of its quantity and collection [5]. And there is no effective model for the problems with fuzziness and randomness in probability theory and multi-objective programming. In this paper: 1) the fuzzy objectives and fuzzy events are represented by triangular fuzzy number; 2) Effect probability model of normal distributions is established and general formulas are also given; 3) we propose the FMOEP model for the multi-objective problems whose objective coefficients are random variables and objectives are fuzzy; 4) Practicability and effectiveness of FMO-EP are proved through a case.

The paper is organized as follows. In the next section, we present the effect probability model of several special fuzzy events that we research in this paper, and some definitions are given. In Section 3, the model of FMO-EP is proposed. Section 4 presents the application of FMO-EP through a product case. Finally, we conclude our paper in section 5. 


\section{Effect probability model of several special fuzzy events}

Probability calculation is the core of decision methods based on probability. In this section, we establish the effect probability calculation of random variable ranging on triangular fuzzy number. For convenience: 1) $(\Omega, \mathscr{B}, P)$ denotes the probability space and $F(\Omega, \mathscr{B})$ denotes the entirety of fuzzy events; 2) $N\left(\mu, \sigma^{2}\right)$ denotes the normal distribution whose parameters are $\mu$ and $\sigma^{2}$ in $(\Omega, \mathcal{B}, P) ; 3) \quad X \sim N\left(\mu, \sigma^{2}\right)$ denotes random variable $X$ subjects to $N\left(\mu, \sigma^{2}\right)$ in $(\Omega, \mathscr{B}, P)$.

Collection and quantity are two properties of fuzzy number as the promotion of interval number. It is always used to represent fuzzy information.

\section{Definition 1 [6]}

Let $A$ be a fuzzy set on real number field $R$ and $A_{\lambda}=\{x \mid A(x) \geq \lambda\}$. If there exist: 1) $A_{1} \neq \varnothing$;2) $A_{\lambda}$ is bounded closed interval for any $\lambda \in[0,1]$; 3) supp $A=\{x \mid A(x)>0\}$ is bounded, then $A$ is called as fuzzy number, the class of fuzzy number is called as fuzzy number space, denotes as $E^{1}$. Especially, if there exist real number $a, b, c$ and satisfy: (1) $A(x)=0$ when $x<a$ or $x>c$; (2) $A(x)=(x-a) /(b-a)$ when $a \leq x<b$; (3) $A(x)=1$ when $b=1$; (4) $A(x)=(c-x) /(c-b)$ when $b<x \leq c$, then $A$ is called as triangular fuzzy number, denoted as $A=(a, b, c)$ for short.

Obviously, the closure of supp $A$ is still closed interval for any $A \in E^{1}$. $A_{0}$ denotes the closure of supp $A$. We propose the effect probability model of fuzzy events based on [2]:

\section{Definition 2}

Let $A \in F(\Omega, B), L(\lambda)$ be the non-continuous function on [0,1] (called as the level effect function), and $\int_{0}^{1} L(\lambda) d \lambda=1$, then

$$
P(A \oplus L(\lambda))=\int_{0}^{1} L(\lambda) P\left(A_{\lambda}\right) d \lambda
$$

is called as the probability of fuzzy event $A$ based on level effect function, and abbreviated as effect probability.

Intuitively speaking, $L(\lambda)$ is a function from $[0,1]$ to $[0,+\infty)$ and it reflects the recognized degree of the level $\lambda$. The larger (smaller) $L(\lambda)$ is, the higher (lower) recognized degree is. Model (1) can effectively reflect the fuzzy decision consciousness of different decision makers.

Combining with model (1) and fuzzy number, we can obtain the following model for the random variable $X$ in $(\Omega, B, P)$ and $A \in E^{1}$,

$$
P(\{X \in A\} \oplus L(\lambda))=\int_{0}^{1} \int_{\underline{a}(\lambda)}^{\bar{a}(\lambda)} L(\lambda) f(x) d x d \lambda .
$$

Here, $f(x)$ is the density function of $X, L(\lambda)$ is the level effect function and $A_{\lambda}=[\underline{a}(\lambda), \bar{a}(\lambda)]$.

Obviously, model (2) is a general significant calculation formula. And there exist the following calculation methods when $f(x)$ is the density function of normal distribution $N\left(\mu, \sigma^{2}\right)$, the level effect function is $L(\lambda)=n \lambda^{n-1}, \quad n \geq 1$ and $A$ is the triangular fuzzy number.

\section{Theorem 1}

Let $X \sim N(0,1)$ in the probability space $(\Omega, \mathscr{B}, P)$, the level effect function $L(\lambda)=n \lambda^{n-1}, n \geq 1$, $A=(a, b, c)$ and $a<b<c$. Then

$$
P(\{X \in A\} \oplus L(\lambda))=\frac{G(a, b, n)}{(b-a)^{n}}+\frac{G(-c,-b, n)}{(c-b)^{n}} .
$$

Here, $G(\alpha, \beta, m)=\int_{\alpha}^{\beta}(x-\alpha)^{m} \cdot \frac{1}{\sqrt{2 \pi}} e^{-\frac{x^{2}}{2}} d x$. 
Proof: According to $A_{\lambda}=[a+(b-a) \lambda, c-(c-b) \lambda]$, the density function $f(x)=\frac{1}{\sqrt{2 \pi}} e^{-\frac{x^{2}}{2}}$ of $N(0,1)$ and the properties of the double integral, we have

$$
\begin{gathered}
P(\{X \in A\} \oplus L(\lambda))=\int_{0}^{1} n \lambda^{n-1} \int_{a+(b-a) \lambda}^{c-(c-b) \lambda} \frac{1}{\sqrt{2 \pi}} e^{-\frac{x^{2}}{2}} d x d \lambda \\
=\int_{a}^{b} \frac{1}{\sqrt{2 \pi}} e^{-\frac{x^{2}}{2}} \int_{0}^{\frac{x-a}{b-a}} n \lambda^{n-1} d \lambda d x+\int_{b}^{c} \frac{1}{\sqrt{2 \pi}} e^{-\frac{x^{2}}{2}} \int_{0}^{\frac{c-x}{c-b}} n \lambda^{n-1} d \lambda d x
\end{gathered}
$$

By above and

$$
\begin{aligned}
\int_{a}^{b} \frac{1}{\sqrt{2 \pi}} e^{-\frac{x^{2}}{2}} \int_{0}^{\frac{x-a}{b-a}} n \lambda^{n-1} d \lambda d x & =\frac{1}{(b-a)^{n}} \int_{a}^{b}(x-a)^{n} \frac{1}{\sqrt{2 \pi}} e^{-\frac{x^{2}}{2}} d x \\
\int_{b}^{c} \frac{1}{\sqrt{2 \pi}} e^{-\frac{x^{2}}{2}} \int_{0}^{\frac{c-x}{c-b}} n \lambda^{n-1} d \lambda d x & =\frac{1}{(c-b)^{n}} \int_{b}^{c}(c-x)^{n} \frac{1}{\sqrt{2 \pi}} e^{-\frac{x^{2}}{2}} d x \\
& =\frac{1}{(c-b)^{n}} \int_{-c}^{-b}(x+c)^{n} \frac{1}{\sqrt{2 \pi}} e^{-\frac{x^{2}}{2}} d x
\end{aligned}
$$

We can obtain the theorem 1.

\section{Corollary 1}

Let $X \sim N\left(\mu, \sigma^{2}\right)$ in the probability space $(\Omega, \mathscr{B}, P)$, the level effect function $L(\lambda)=n \lambda^{n-1}, n \geq 1, A=(a, b, c)$ and $a<b<c$. Then

\section{Corollary 2}

$$
P(\{X \in A\} \oplus L(\lambda))=\frac{\sigma^{2} G\left(\frac{a-\mu}{\sigma}, \frac{\mu-b}{\sigma}, n\right)}{(b-a)^{n}}+\frac{\sigma^{2} G\left(\frac{\mu-c}{\sigma}, \frac{\mu-b}{\sigma}, n\right)}{(c-b)^{n}} .
$$

Let $X \sim N\left(\mu, \sigma^{2}\right)$ in the probability space $(\Omega, \mathscr{B}, P)$, the level effect function $L(\lambda)=n \lambda^{n-1}, n \geq 1$ and $a<b<c$. Then

$$
\begin{aligned}
& P(\{X \in(a, b, b)\} \oplus L(\lambda))=\frac{\sigma^{2} G\left(\frac{a-\mu}{\sigma}, \frac{\mu-b}{\sigma}, n\right)}{(b-a)^{n}}, \\
& P(\{X \in(b, b, c)\} \oplus L(\lambda))=\frac{\sigma^{2} G\left(\frac{\mu-c}{\sigma}, \frac{\mu-b}{\sigma}, n\right)}{(c-b)^{n}} .
\end{aligned}
$$

Remark 1 In reality, randomness and fuzziness always occur together in the decision problems (such as: one factory makes a sales goal "not less than 1000 as far as possible". In this question, the demand is a variable subjecting to some probability distribution and the objective is a fuzzy event). And "not less (more) than the giving value $b$ " is the most common form in multi-objective problems. So if $X$ denotes the random variable, $B$ denotes fuzzy events "not less than $b$ ", then the probability of $B$ can be denoted as (here, $L(\lambda)$ is the level effect function; $\delta$ is the highest limit less than $b)$ :

$$
P(\{X \in B\} \oplus L(\lambda))=P(X \in(b-\delta, b, b) \oplus L(\lambda))+P(X \geq b) .
$$

It is the theoretical support for the multi-objective programming. So the calculation can be used to dealing with the probability calculation of fuzzy events in multi-objective problems.

\section{The model of FMO-EP}

Multi-objective programming as a common decision method which deals with complex system optimization, has successfully applied to design process of new products, investment analysis and many other fields. The general model of multi-objective programming is as following: 


$$
\left\{\begin{array}{l}
\min f=\left\{f_{1}(x), f_{2}(x), \cdots, f_{t}(x)\right\} \\
\text { s.t. } \quad g_{i}(x) \leq 0, i=1,2, \cdots, m
\end{array} .\right.
$$

Here, $f_{t}(x)$ is the objective function of objective $t, x=\left\{x_{1}, x_{2}, \cdots, x_{n}\right\} \in R^{n}$ is the decision vector.

Model (8) is a abstract model of multi-objective problem in the certain environment. It has a widely practicability but exists no calculation method. Then goal programming was firstly put forward and appointed that they were absolutely priority relation among the objectives in 1961 [7]. Achieving the high level priority objective, the next objective can be solved step by step.

But it is not practical for absolutely priority relation among the objectives in the multi-objective programming. For this problem, some others proposed the following model [8]:

$$
\begin{cases}\min & \Sigma\left[f_{1}(x), f_{2}(x), \cdots, f_{t}(x)\right] \\ \text { s.t. } & g_{i}(x) \leq 0, i=1,2, \cdots, m\end{cases}
$$

Here, $\Sigma\left[f_{1}(x), f_{2}(x), \cdots, f_{t}(x)\right]$ is the comprehensive function of $t$ objectives, $f_{t}(x)$ is the objective function of objective $t, x=\left\{x_{1}, x_{2}, \cdots, x_{n}\right\} \in R^{n}$ is the decision vector.

Obviously, model (8) and (9) are the general multi-objective programming in the certain environment. But the fuzziness along with the randomness is common in the multi-objective programming. Such as: one enterprise decides to invest and product $m$ independent products, the cost and the profit of per production are random variables $\xi_{i}, \eta_{i}$ respectively. And the invest objectives: $M_{1}$, the total investment amount $\mathrm{Y}_{1}$ is less than $a$ thousand yuan as far as possible. There exists $Y_{1}=\sum_{i=1}^{n} \xi_{i} x_{i}, \xi_{i} \sim N\left(\mu_{i}, \sigma_{i}^{2}\right), Y_{1} \sim N\left(\sum_{i=1}^{n} \xi_{i} x_{i}, \sum_{i=1}^{n} x_{i}^{2} \sigma_{i}^{2}\right)$.The objectives are fuzzy and the decision environment is random. Combining with the discussion in the second section, we propose the FMO-EP in random environment:

$$
\begin{cases}\max & f=\left\{P_{1}\left(\left(Y_{1} \in M_{1}\right) \oplus L(\lambda)\right), \cdots, P_{t}\left(\left(Y_{t} \in M_{t}\right) \oplus L(\lambda)\right)\right\} \\ \text { s.t. } & g_{i}(x) \leq 0, i=1,2, \cdots, m\end{cases}
$$

Here, $M_{i}, P_{i}$ denote the objective $i$ and its effect probability, $Y_{t}$ is the objective function of objective $t$.

Model (10) is a general multi-objective programming for fuzzy objectives in random environment. Not only the satisfactory solution but also the probability of every decision objective can be solved. We also can solve the model (10) with the traditional multi-objective decision making methods (such as linear weighted method, ideal point method, target and many other methods). Further, narrowing the scope of the feasible region and simplifying the solving process by introducing the probability constrains. Beside, model (10) reflects the decision consciousness of different decision makers. And the decision consciousness of decision makers play a key role in the decision process.

In this part, we present the FMO-EP for the case of the coefficients are random variables in the objective functions and the objectives are fuzzy. This model can successfully solve the fuzziness and the randomness together. And this kind question is common in the decision analysis, management decision and many other fields.

\section{The application of FMO-EP}

Case description: An enterprise decides to produce product $A$ and $B$. Through investigation and analysis, we have the following information: The cost of per production of $A$ and $B$ are random variables $\xi_{1}, \xi_{2}$ subject to $N(2100,200)$ and $N(4800,500)$ respectively; The profit of per production of $A$ and $B$ are random variables $\eta_{1}, \eta_{2}$ subject to $N(3600,400)$ and $N(6500,800)$ respectively; the total demand for $A$ and $B$ is no less than 9 tons. The largest production capacity of $A$ and $B$ are 5 tons and 8 tons. There are two objectives for this: $M_{1}$, the total investment 
amount $Y_{1}$ per month is less than 30 thousand yuan as far as possible, 33 thousand is the maximum we can accept: $M_{2}$, the total revenue $Y_{2}$ is more than 45 thousand yuan as far as possible, and 40 thousand is the minimum we can accept. The weights of the two objectives are 0.4 and 0.6 respectively. The level effect function $L(\lambda)=2 \lambda$.

Obviously, this is a fuzzy multi-objective programming in the random environment. The objective 1) and 2) are both fuzzy and random because the $\xi_{1}, \xi_{2}, \eta_{1}, \eta_{2}$ are random variables. If the throughput of $A$ and $B$ per mouth is $x_{1}, x_{2}$ respectively, then there exist:

$$
\left\{\begin{array}{l}
Y_{1}=\xi_{1} x_{1}+\xi_{2} x_{2}, \\
Y_{1}=\eta_{1} x_{1}+\eta_{2} x_{2} .
\end{array}\right.
$$

Further, $Y_{1} \sim N\left(2100 x_{1}+4800 x_{2}, 200 x_{1}^{2}+500 x_{2}^{2}\right)$ and $Y_{2} \sim N\left(3600 x_{1}\right.$

$\left.+6500 x_{2}, 400 x_{1}^{2}+800 x_{2}^{2}\right)$. And the objectives are fuzzy, so we can establish the following model by the FOM-EP model (10):

$$
\begin{cases}\max & \left.f=0.6 P_{1}\left(\left(Y_{1} \in M_{1}\right) \oplus L(\lambda)\right)+0.4 P_{2}\left(\left(Y_{2} \in M_{2}\right) \oplus L(\lambda)\right)\right\} \\ \text { s.t. } & 0 \leq x_{1} \leq 5 \\ & 0 \leq x_{2} \leq 8 \\ & x_{1}+x_{2} \geq 9\end{cases}
$$

There is no analytical method for the model (12). But we get the result that $4.8749,4.2072$ are the production quantity of $A$ and $B$ that the enterprise expected, respectively by genetic algorithm. Model (12) reflects the different consciousness of different decision makers by the corresponding parameter value. And it can narrow the scope of the feasible region and simplify the solving process by introducing the probability constrains.

\section{Conclusions}

In this paper, we present the general formula of effect probability that random variable rang on the fuzzy number based on the continuous distribution. FMO-EP is also proposed in the random environment for the multi-objective programming problems whose objective coefficients are random variables and objectives are fuzzy. Theoretical analysis and practice show that: 1) fuzzy number is a useful tool describing the fuzzy information. 2) effect probability has good calculation properties and valuable application and it reflects the fuzzy decision consciousness. 3) FMO-EP model effectively solves the questions in the random environment. 4) The effect probability on some other distributions and fuzzy multi-objective programming are the further research.

\section{Acknowledgements}

The research work was supported by the National Natural Science Foundation of China under Grant No. 71371064 and Natural Science Foundation of Hebei Provincial under Grant No. F2015208100 and Grant No. F2015208099.

\section{References}

[1] Zadeh, L.A., Probability measures of fuzzy events. Mathematic Analysis and Applications, 23, pp. 421-427, 1968.

[2] Chen, D. G., Yang, W. X. \& Li, F. C., Measures of general fuzzy rough sets on a probabilistic space . Information Sciences, 178, pp. 3177-3182, 2008.

[3] Hulsurkar, S., Biswal M. P. \& Sinha S. B., Fuzzy programming approach to multi-objective stochastic linear programming problems. Fuzzy Sets and Systems, 88, pp. 173-181, 1997. 
[4] Abdelaziz, F. B., Aouni, B. \& Fayedh, R. E., Multi-objective stochastic programming for portfolio selection. European Journal of Operational Research, 177, pp. 1811-1823, 2007.

[5] Merigo, J.M., Fuzzy decision making with immediate probabilities. Computers \& Industrial Engineering, 58, pp. 651-657, 2010.

[6] Hu, B.Q., The basis of fuzzy theory. Wuhan: Wuhan University Press, 2010.

[7] Yu, C.T., Li, F.C. \& Hui, H.Q., Operations research. Beijing: Science Press, 2011. 